\title{
Note on holographic subregion complexity and QCD phase transition
}

\author{
Mahsa Lezgi ${ }^{*}$ and Mohammad Ali-Akbari ${ }^{\dagger}$ \\ Department of Physics, Shahid Beheshti University G.C., Evin, Tehran 19839, Iran
}

(Received 25 October 2019; published 29 January 2020)

\begin{abstract}
Using holographic subregion complexity, we study the confinement-deconfinement phase transition of a QCD-like gauge theory, holographically. In the model we consider here, we observe a connection between the potential energy of a probe meson and the behavior of its complexity. Moreover, near the critical point, at which the phase transition takes place, our numerical calculations indicate that we need less information to specify a meson in the nonconformal vacuum than in the conformal one, despite the fact that the nonconformal vacuum has larger energy.
\end{abstract}

DOI: 10.1103/PhysRevD.101.026022

\section{INTRODUCTION}

Gauge-gravity duality, or more generally the holographic idea, has provided a new framework to investigate various properties of nonperturbative theories within the past two decades. This duality is, in fact, a strong-weak duality and maps a strongly coupled quantum gauge field theory to a weakly coupled classical gravity in a higher dimension. This idea has been frequently applied to describe various phenomena in strongly coupled field theories, for which the standard perturbation method is not applicable, ranging from condensed matter physics to low-energy quantum chromodynamics (QCD), the theory of strong interactions $[1,2]$. Central aspects of low-energy QCD such as the confined phase, confinement-deconfinement phase transition, and chiral symmetry breaking have been discussed within the concept of gauge-gravity duality; for instance, see [2] and references therein.

One of the interesting areas of theoretical physics is quantum information theory, and, according to the holographic idea, an outstanding connection has been developed between quantum information and gravity started by the Hubney-Ryu-Takayanagi proposal for entanglement entropy, defined as a measure of the quantum correlation of a pure quantum state. Entanglement entropy is one of the important quantities in the context of information theory; fortunately, there exists a simple geometrical prescription to describe it and its properties [3,4], and it passes many tests successfully.

\footnotetext{
s_lezgi@sbu.ac.ir

m_aliakbari@sbu.ac.ir
}

Published by the American Physical Society under the terms of the Creative Commons Attribution 4.0 International license. Further distribution of this work must maintain attribution to the author(s) and the published article's title, journal citation, and DOI. Funded by SCOAP .
Another main concept in information theory is quantum complexity. It is defined as the minimum number of unitary operators needed to prepare a target state from a reference state or, in other words, the difficulty in converting one state to another one $[5,6]$. Describing complexity holographically has received a lot of interest in the literature these days, and there exist two conjectures, namely, CV $($ complexity $=$ volume $)$ and CA (complexity $=$ action). In the CA conjecture, the complexity is given by the bulk action evaluated on the Wheeler-de Witt patch anchored at some boundary time $[7,8]$. The $\mathrm{CV}$ proposal states that the complexity is identified as the volume of the extremal or maximal volume of a codimensional-one hypersurface $\mathcal{B}$ in the bulk ending on a time slice of the boundary

$$
\mathcal{C}=\frac{V(\mathcal{B})}{\hat{L} G_{N}}
$$

where $G_{N}$ is the five-dimensional gravitational constant and $\hat{L}$ is some length scale of the bulk, for example, the antide Sitter (AdS) radius. Inspired by the Hubney-RyuTakayanagi proposal, this conjecture for the whole boundary system is generalized for subsystems [9]. The complexity for a subsystem $A$ on the boundary equals the volume of the codimensional-one hypersurface enclosed by Hubney-Ryu-Takayanagi surface $\gamma_{A}$ :

$$
\mathcal{C}_{A}=\frac{V_{\gamma_{A}}}{8 \pi R G_{N}}
$$

where $R$ is the $\mathrm{AdS}_{5}$ radius and $\mathcal{C}_{A}$ is known as holographic subregion complexity (HSC). Some recent works on CA and CV prescriptions and HSC for different gravity models can be found in Refs. [10-20]. In this paper, we would like to compute HSC to study the confinement-deconfinement phase transition in a QCD-like gauge theory, and the questions we are indeed interested in are, is HSC a relevant order parameter of the phase transition or can HSC 
recognize the favorable vacuum, or, more generally, what do we learn about the phase transition using HSC as a quantity we can calculate? To do so, we consider a background which almost perfectly describes the potential in the confined phase [21]. Moreover, using entanglement entropy, the critical energy scale $\Lambda_{c}$, the scale at which the confinement-deconfinement phase transition takes place, can be excellently found in this background [22]. Thus, we start introducing the background(s) and then compute the HSC on different relevant background(s) and discuss its properties to answer the above questions.

\section{BACKGROUNDS}

In Ref. [21], a five-dimensional metric, called modified AdS (MAdS), is defined as

$$
d s^{2}=\frac{R^{2}}{z^{2}} g(z)\left(-d t^{2}+d \vec{x}^{2}+d z^{2}\right),
$$

and the black hole version of the above metric, which we call modified black hole (MBH), is introduced by [23]

$$
d s^{2}=\frac{R^{2}}{z^{2}} g(z)\left(-f(z) d t^{2}+d \vec{x}^{2}+\frac{d z^{2}}{f(z)}\right),
$$

where $g(z)=e^{(c / 2) z^{2}}, \vec{x} \equiv\left(x_{1}, x_{2}, x_{3}\right)$, and $z$ is the radial coordinate. $c$ is a modifier parameter with energy ${ }^{2}$ dimension. The above backgrounds are dual to QCD-like gauge theories at zero and nonzero temperature. Therefore, from the fit to the slope of the Regge trajectories, it is estimated to be $0.9 \mathrm{GeV}^{2}$ [21]. This number can be found more precisely by determining the critical energy scale, and it turns out to be $0.94 \mathrm{GeV}^{2}$ [22]. Clearly, the backgrounds (3) and (4) are asymptotically $\mathrm{AdS}_{5}$ with radius $R$, and the holographic QCD-like model is living on the boundary of the backgrounds located at $z=0$.

The background (4) is a natural extension of (3) for including thermodynamics where $f(z)=1-z^{4} / z_{h}^{4}$. The Hawking temperature is given by $T=\frac{1}{\pi z h}$, and $z_{h}$ is the position of the horizon. By setting modifier parameter $c$ equal to zero in backgrounds (3) and (4), one can easily find the $\mathrm{AdS}_{5}$ background (AdS) and $\mathrm{AdS}_{5}$ planar black hole metric $(\mathrm{BH})$, respectively. The phase transition, in the gravitational picture, is described by changing the background geometry from (3) to (4), and we will see that HSC does confirm it near the critical point. More properties of these geometries have been discussed in Refs. [21-24].

\section{POTENTIAL ENERGY}

In the quantum field theory, potential energy between a quark and antiquark (meson) can be obtained from the expectation value of the rectangular Wilson loop, as a nonlocal gauge-invariant operator. In fact, it can be done by evaluating the expectation value of the Wilson loop on a

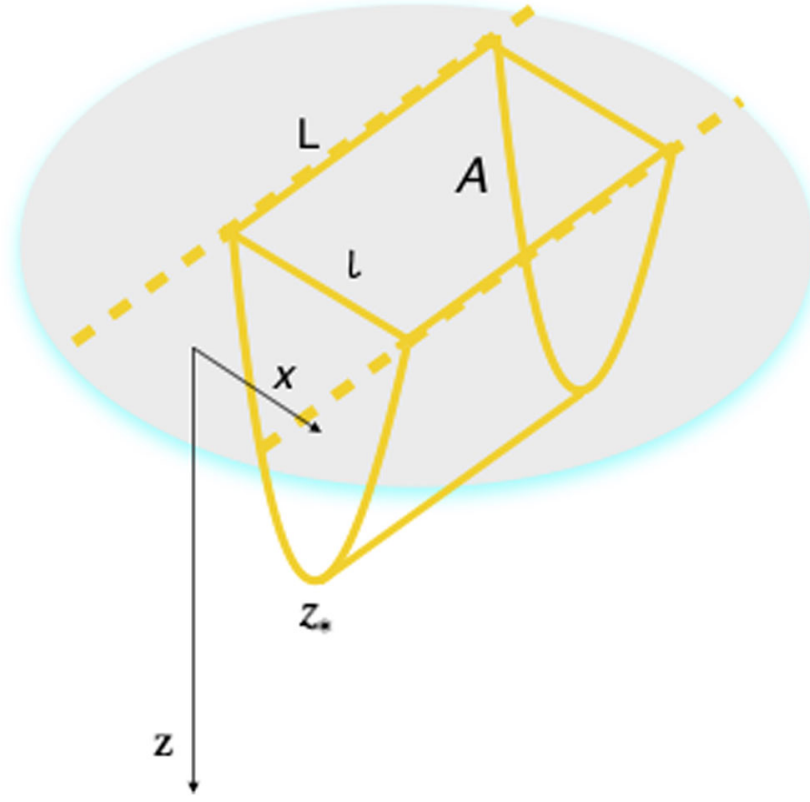

FIG. 1. A strip entangling surface of length $l$ and width $L \rightarrow \infty$.

rectangular loop, $\mathcal{R}$ with two sides, time $\mathcal{T}$, and distance $r$, that $\mathcal{T} \gg r$ (see Fig. 1 by the replacement $L \rightarrow \mathcal{T}$ and $l \rightarrow r$ ). This configuration is equivalent to a static quarkantiquark pair with the distance $r$ between them. It is well known that the expectation value of the Wilson loop is dual to the on-shell classical action $S(\mathcal{R})$ of a classical string whose world sheet ends on the $\mathcal{R}$, rectangular loop at the boundary [25]. It is then straightforward to find potential energy corresponding to the binding energy of the pair in the background (3), and it turns out [21]

$$
V(r)= \begin{cases}p\left(-\frac{\kappa_{0}}{r}+\sigma_{0} r+O\left(r^{3}\right)\right), & r \rightarrow 0, \\ p(\sigma r), & r \rightarrow \infty,\end{cases}
$$

where $p \approx 0.94, \kappa_{0} \approx 0.23, \sigma_{0} \approx 0.16 \mathrm{GeV}^{2}$, and $\sigma \approx$ $0.19 \mathrm{GeV}^{2}$ for $c=0.9 \mathrm{GeV}^{2}$. This potential is similar to the Cornell potential and gives the expected linear and $1 / r$ behavior at a large and short distance, respectively. In other words, this background describes the QCD at a low-energy, confined phase. For more details, see Ref. [21].

\section{ENTANGLEMENT ENTROPY}

A well-known nonlocal observable in the information theory is entanglement entropy [26]. Consider a quantum field theory whose pure state is described by the density matrix $\rho$. The entanglement entropy of a spatial subregion $A$, with complement $\bar{A}$, denotes how much entanglement exists between $A$ and $\bar{A}$, and it is given by

$$
S_{A}=-\operatorname{Tr}\left(\rho_{A} \log \rho_{A}\right),
$$

where $\rho_{A}=\operatorname{Tr}_{\bar{A}}(\rho)$ is the reduced density matrix obtained by tracing over the degrees of freedom in the region $\bar{A}$. The 

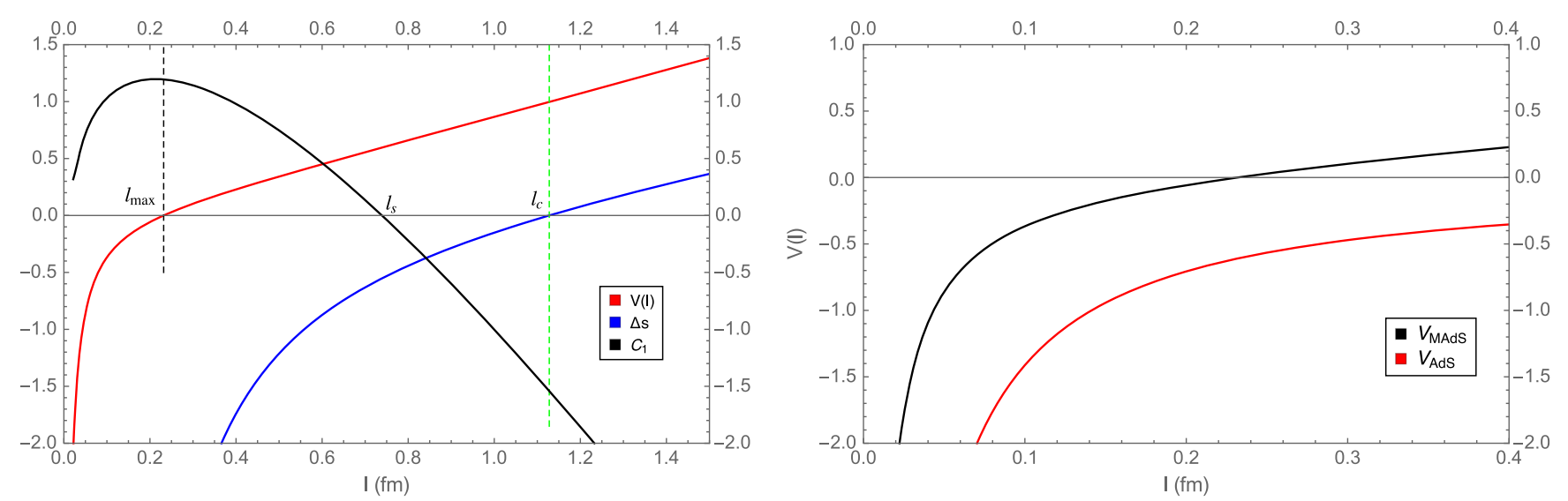

FIG. 2. Left: The potential energy $V(l)$, the difference of the entanglement entropies $\Delta S$ and $C_{1}$ in terms of $l(\mathrm{fm})$ for $c=0.94 \mathrm{GeV}{ }^{2}$. Right: The potential of meson in AdS and MAdS background for $c=0.94 \mathrm{GeV}^{2}$. Note that $1 \mathrm{fm}=(197.3 \mathrm{MeV})^{-1}=5.068 \mathrm{GeV}^{-1}$.

entanglement entropy of $A$ shows the amount of information lost when an observer is limited to the subregion $A$. Although calculating the entanglement entropy is normally difficult, the holographic method provides a handy approach to obtain the entanglement entropy. In fact, Ryo and Takayanagi first proposed in Ref. [3] that the entanglement entropy can be computed from

$$
S_{A}=\frac{\operatorname{area}\left(\gamma_{A}\right)}{4 G_{N}},
$$

where $\gamma_{A}$ is a codimension-2 minimal surface whose boundary $\partial \gamma_{A}$ coincides with the boundary of the subregion $A$ on the boundary of the bulk where the quantum field theory lives, i.e., $\partial \gamma_{A}=\partial A$. This proposal received a lot of interest during the past decade and passed several nontrivial checks known in the quantum field theory. For more details, we refer the interested reader to Ref. [4].

Now let us consider a strip entangling surface of length $l$ and width $L \rightarrow \infty$. Indeed, the subsystem $A$ is defined by $-\frac{l}{2}<x_{1}(\equiv x)<\frac{l}{2}$ and $x_{2}, x_{3} \in(-\infty,+\infty)$ at a given time; see Fig. 1. Entanglement entropy is proportional to the minimal area of $\gamma_{A}$, and it turns out to be [22]

$$
S_{A}^{(c)}=\frac{L^{2}}{2 G_{5}} \int_{0}^{z_{*}} \frac{R^{3} z^{3} g(z)^{3}}{z_{*}^{3} \sqrt{z_{*}^{6} g(z)^{3}-z^{6} g\left(z_{*}\right)^{3}}} d z,
$$

where $z_{*}=z(x=0)$. Considering the disconnected configuration described by two surfaces located at $x= \pm l / 2$, the entanglement entropy for the disconnected case becomes [22]

$$
S_{A}^{(d)}=\frac{L^{2}}{2 G_{5}} \int_{0}^{\sqrt{2 / c}}\left(\frac{R^{2} g(z)}{z^{2}}\right)^{3 / 2} d z
$$

Then, in order to specify the phase transition, according to the results reported in Ref. [22], we define

$$
\Delta S(l) \equiv \frac{2 G_{5}}{L^{2}}\left(S_{A}^{(c)}-S_{A}^{(d)}\right) .
$$

Using the above definition, the phase transition and its critical energy scale have been predicted [22]. It is shown that the phase transition occurs at $l_{c} \approx 1 \mathrm{fm}$ (compatible with the size of the hadrons) for a reasonable value of $c$, i.e., $c \approx 0.9 \mathrm{GeV}^{2}$. As shown in Fig. 2 (blue curve), the point at which the sign of $\Delta S$ changes characterizes the phase transition. It occurs in the regime of $l$ for which the linear behavior of the Cornell-type potential has dominated.

\section{SUBREGION HOLOGRAPHIC COMPLEXITY}

Motivated by holographic entanglement entropy and by an extension of the Hubney-Ryu-Takayanagi proposal, HSC for a subsystem $A$ in the boundary theory is defined as follows [9]:

$$
\mathcal{C}_{A}=\frac{V_{\gamma_{A}}}{8 \pi R G_{5}},
$$

where $V_{\gamma_{A}}$ is the volume of the codimension-one hypersurface enclosed by minimal hypersurface $\gamma_{A}$ obtained to calculate holographic entanglement entropy. Then, using metric (3), one easily finds the area of the minimal surface:

$$
S=\frac{L^{2}}{4 G_{5}} \int_{-l / 2}^{l / 2} \frac{R^{3}}{z^{3}} g(z)^{3 / 2} \sqrt{1+z^{\prime}(x)^{2}} d x,
$$

where $z(x)$ [or, equivalently, $x(z)$ ] is the profile of the minimal surface. Then, by using the constant of motion, the profile of the minimal surface is obtained:

$$
x(z)=2 \int_{z}^{z_{*}} \frac{z^{3} g\left(z_{*}\right)^{3 / 2}}{\sqrt{z_{*}^{6} g(z)^{3}-z^{6} g\left(z_{*}\right)^{3}}} d z .
$$

In the static case, the volume enclosed by $\gamma_{A}$ is obtained by integrating the inside of the minimal surface. It can be 
done by slicing the bulk with planes of constant $z$. We therefore have

$$
V_{\gamma_{A}}\left(z_{*}\right)=2 L^{2} \int_{0}^{z_{*}} \frac{R^{4}}{z^{4}} g(z)^{2} x(z) d z
$$

and a similar calculation for the metric (4) leads to

$$
V_{\gamma_{A}}\left(z_{*}\right)=2 L^{2} \int_{0}^{z_{*}} \frac{R^{4}}{z^{4}} \frac{g(z)^{2} x(z)}{\sqrt{f(z)}} d z .
$$

It is obvious that the above two equations reduce to the case of AdS and BH by setting $c=0$, respectively. Similar to the case of entanglement entropy, the volume is divergent, and we need to introduce a normalized volume, relative complexity, as follows:

$$
\begin{aligned}
C_{1} & \equiv \frac{\mathcal{C}_{\mathrm{MAdS}}}{\mathcal{C}_{\mathrm{AdS}}}-1, \\
C_{2} & \equiv \frac{\mathcal{C}_{\mathrm{MBH}}}{\mathcal{C}_{\mathrm{BH}}}-1,
\end{aligned}
$$

where $\mathcal{C}_{\mathrm{MAdS}}, \mathcal{C}_{\mathrm{AdS}}, \mathcal{C}_{\mathrm{MBH}}$, and $\mathcal{C}_{\mathrm{BH}}$ are the HSC for $A$ in MAdS, AdS, MBH, and BH geometry, respectively. We use the above definitions to discuss the phase transition.

\section{NUMERICAL RESULTS}

In this section, we will argue that our findings from the numerical calculations of HSC at zero as well as nonzero temperature are reasonable. Indeed, a general expectation is that the HSC should behave differently at zero and nonzero temperatures, since each of them indicates the confinement and nonconfinement regime in field theory, respectively.

\section{A. Zero temperature}

As was previously mentioned, the state we consider here corresponds to the modified background (3) probed by a classical string (quark-antiquark pair or meson in the dual field theory) on the gravity side. Therefore, according to the definition (11), $\mathcal{C}_{\text {MAdS }}\left(\mathcal{C}_{\text {AdS }}\right)$ is identified with the complexity of the probe meson living in a nonconformal (conformal) vacuum both at zero temperature. Our results are shown in Fig. 2 (left). The red and blue curves denote the potential energy between the pair and difference of entanglement entropies $\Delta S$, respectively. The black curve is the relative complexity of the pair obtained from Eq. (16a). The horizontal axis is the distance between the quark-antiquark pair, i.e., $r \equiv l$. As is clearly seen, the relative complexity increases with $l$ up to a maximum at $l=l_{\max }$ and then decreases with rising $l$. It is important to notice that it changes sign, say, at $l=l_{s}$. There are obviously three different regions as follows: (i) $C_{1}>0$ and $l<l_{s} .-\mathcal{C}_{\text {MAdS }}>\mathcal{C}_{\text {AdS }}$ for small values of $l$, i.e., $l<l_{s}$, probing the UV regime in field theory. To be more specific, at a high energy the information needed to prepare the state of a meson in the nonconformal vacuum is larger than in the conformal one.

(ii) $C_{1}<0$ and $l_{s}<l<l_{c}$. $-\mathcal{C}_{\text {MAdS }}<\mathcal{C}_{\text {AdS }}$, and, therefore, the state of a meson is easier to specify in the nonconformal vacuum than the other one.

(iii) $C_{1}<0$ and $l>l_{c}$. - It is similar to the previous case.

These above categories have a simple physical interpretation as follows. Based on results reported in Ref. [22], the QCD phase transition happens in the region with $C_{1}<$ 0 and exactly at $l=l_{c}$ and $\Lambda_{c}=l_{c}^{-1}=175 \mathrm{MeV}$. Therefore, the region with $l<l_{c}$ describes the field theory in the confined phase, the region of $l>l_{c}$ indeed is not physical, and the background (3) must be replaced with the black hole one which, in this case, is (4) or an $\mathrm{AdS}_{5}$ planar black hole. As a result, the third category is not acceptable. Moreover, for allowed values of $l$ and near $l_{c}$ in the confined phase, the second category, the complexity of the meson in the nonconformal vacuum is less than the vacuum one, or, equivalently, we need less information to prepare the meson in the nonconformal vacuum. It seems reasonable, since the background (3) describes the confined phase of QCD and produces the relevant potential energy between the pair given by (5) in agreement with our intuition. Put in other words, although HSC does not help us to find the value of critical energy scale $\Lambda_{c}$, it states that for $\Lambda\left(=l^{-1}\right) \lesssim \Lambda_{c}$ the state of the meson in the nonconformal vacuum is easier to specify.

About the first category, we would like to make a comment on the potential energy. It can be seen from this figure that the potential energy of the meson becomes zero around $l_{\max }$, at which the maximum value of the relative complexity $C_{1}$ happens. Furthermore, it is significant to notice that the difference between conformal and nonconformal vacuums is expected to realize more by larger values of $l$, and, as a result, the relative complexity should be a monotonically increasing function. However, when the $r$ term in the potential starts dominating, the relative complexity $C_{1}$ decreases. Therefore, one may conclude that at zero temperature, as soon as the $r\left(\frac{1}{r}\right)$ term is dominant in the potential energy, the relative complexity decreases (increases). In fact, at least in this model, the increase or decrease of relative complexity $C_{1}$ seems to be related to the dominant term in the potential energy.

Our results become more interesting when we consider the stability of a meson at zero temperature in a conformal and nonconformal vacuum, too. The potential energies for both cases have been plotted in figure 2, right. As this figure shows, a meson in the nonconformal vacuum is always less stable than the conformal one. Moreover, our 
TABLE I. A short summary of information and stability in a nonconformal vacuum comparing to a conformal one.

\begin{tabular}{lcc}
\hline \hline Cases & Information & Stability \\
\hline$T=0, l<l_{s}$ & More & Less \\
$T=0, l_{s}<l<l_{c}$ & Less & Less \\
$T \neq 0, l>l_{c}$ & More & More \\
\hline \hline
\end{tabular}

complexity calculation indicates that the less stable meson needs less information to specified, of course, near the critical point, $175 \mathrm{MeV} \lesssim E \lesssim 270 \mathrm{MeV}$. It therefore seems that near the critical point a better option is to choose the less stable meson (in a nonconformal vacuum) with less information. However, for $l<l_{s}$ the story is vice versa, and the less stable meson needs more information to be specified; see Table I. One may thus conclude that the regime of $E>270 \mathrm{MeV}$ is much better described by a conformal vacuum (instead of a nonconformal one), since it is more stable and needs less information to specify a meson.

We would like to mention that we intuitively expect that more (less) information is needed to specify a more (less) stable meson. Altogether, the only conflict arises when $E>270 \mathrm{MeV}$, in the region where the $\frac{1}{r}$ term dominates or $r$ term starts dominating in the potential. Then this incompatibility is cured in the regime that the $r$ term in the potential plays a more important role, i.e., close to the critical point. As a matter of fact, it seems that the $r$ term in the potential help us to find the appropriate result corresponding to our intuition.

Since complexity refers to classifying various quantum states based on their difficulties, it can be also defined as a difficulty in creating a state [5]. From this point of view, the above results indicate that, near the critical temperature, the difficulty in preparing a meson state in the nonconformal vacuum, dual to (3), is less than the conformal.

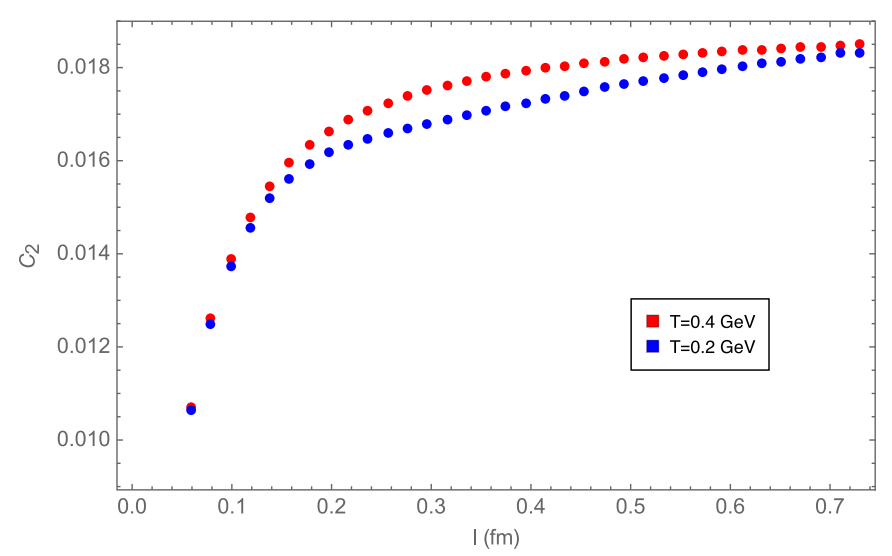

\section{B. Finite temperature}

Similar to the zero temperature case, we plot $C_{2}$ in terms of $l$ in Fig. 3 (left). We observe that there is no substantial difference among various temperatures and also $C_{2}$ is always positive, i.e., $\mathcal{C}_{\mathrm{MBH}}>\mathcal{C}_{\mathrm{BH}}$. Therefore, in order to specify the state of the meson in the thermal vacuum, less information is needed than in the nonconformal thermal vacuum. It is easy to check that, for a given value of $l$, a quark and antiquark pair is less bounded in the thermal vacuum, as one can see in Fig. 3 (right), and, thus, the bound state in the thermal nonconformal vacuum is stronger. In other words, the color screening, which prevents the quark and antiquark from binding to each other in the deconfined phase, is stronger in the thermal vacuum. Therefore, in order to specify a bound state in the thermal nonconformal vacuum, we need more information, since the quark and antiquark are more bounded.

We would also like to mention that, based on Fig. 2 and its discussion, the potential energies in Fig. 3 (right) are obviously not linear, we naively expect the relative complexity $C_{2}$ increases similar to Fig. 2, and it is confirmed by Fig. 3 (left). Therefore, it seems that in a given vacuum a connection between the complexity of a meson and the potential energy of the pair exists. In fact, it can be classified in two categories as follows:

(i) When the linear term is not as important as the $r$ term in the potential energy, the absolute value of relative complexity increases. This result is confirmed by the relative complexity $C_{1}$ and $C_{2}$. Note that although, for instance, the relative complexity $C_{2}$ is not negative, this positivity has no physical interpretation, because one can define $C_{2}^{\prime} \equiv \frac{\mathcal{C}_{\mathrm{BH}}}{\mathcal{C}_{\mathrm{MBH}}}-1$, which is clearly negative and leads to the same results. Moreover, it seems that the place of the maximum (or minimum) depends on the strength of the potential energies, though we do not have any analytical or numerical calculation.

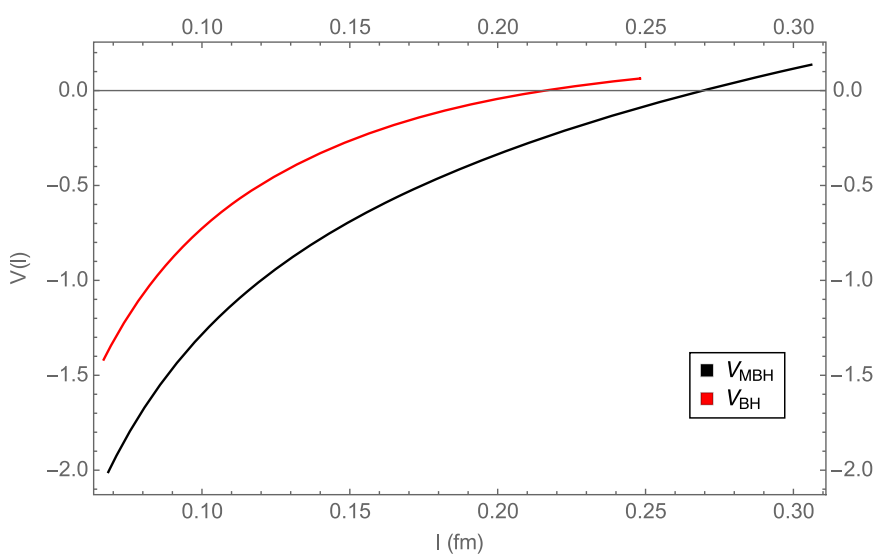

FIG. 3. Left: $C_{2}$ as a function of $l(\mathrm{fm})$ for $c=0.94 \mathrm{GeV}^{2}$. Right: The potential energy of meson for $\mathrm{BH}$ and $\mathrm{MBH}$ for $T=200 \mathrm{MeV}$. 


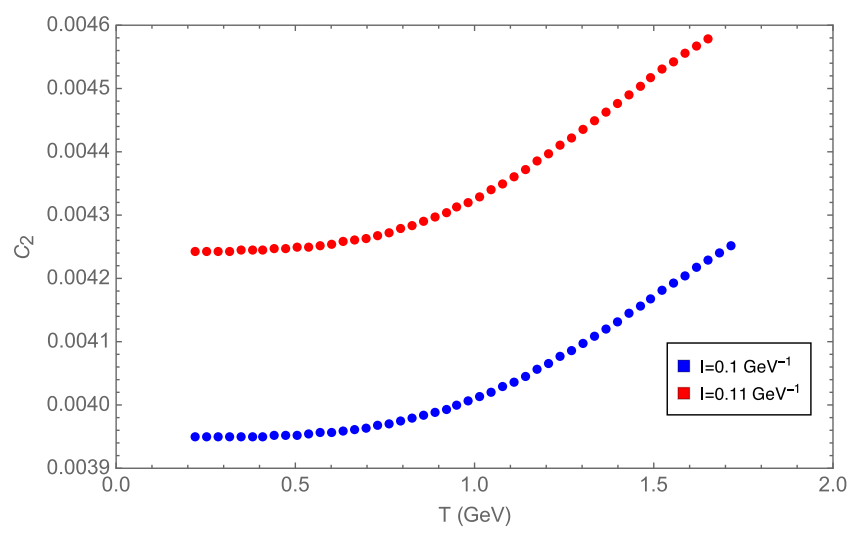

FIG. 4. $C_{2}$ as a function of $T$ for $c=0.94 \mathrm{GeV}^{2}$.

(ii) Otherwise, when the $r$ term is dominant, the absolute value of relative complexity decreases.

In Fig. 4 , we plot $C_{2}$ in terms of the temperature for two values of $l$ to cross-check our previous result about the color screening. It is obvious that for larger values of $l$ we need more information to specify the state of the probe meson in a nonconformal thermal vacuum. In other words, the screening becomes stronger when the temperature or distance $l$ is raised. Therefore, as we discussed already, the screening of the static quark-antiquark potential and $C_{2}$ together increase.

Before closing this section, we would like to note that using Fig. 4 it is impossible to specify the dependence of the complexities $\mathcal{C}_{\mathrm{MBH}}$ or $\mathcal{C}_{\mathrm{BH}}$ on the temperature [27]. Indeed, for $T \gtrsim \Lambda_{c}$ the relative complexity $C_{2}$ is almost constant, and, therefore, both complexities $\mathcal{C}_{\mathrm{MBH}}$ and $\mathcal{C}_{\mathrm{BH}}$ can be an increasing or decreasing function of the temperature or even independent of $T$. The same argument is also true for other values of the temperature. However, using this figure at a fixed temperature, it is realized that $\frac{\mathcal{C}_{\mathrm{MBH}}}{\mathcal{C}_{\mathrm{BH}}} \propto T$ for large enough temperatures, meaning that the meson complexity in a nonconformal vacuum is always bigger than a conformal one.

\section{ACKNOWLEDGMENTS}

M. A. thanks CERN TH-Division for warm hospitality.
[1] J. Casalderrey-Solana, H. Liu, D. Mateos, K. Rajagopal, and U. A. Wiedemann, Gauge/string duality, hot QCD and heavy ion collisions, arXiv:1101.0618.

[2] M. Ammon and J. Erdmenger, Gauge/Gravity Duality: Foundations and Applications (Cambridge University Press, Cambridge, England, 2015).

[3] T. Nishioka, S. Ryu, and T. Takayanagi, Holographic entanglement entropy: An overview, J. Phys. A 42, 504008 (2009).

[4] M. Rangamani and T. Takayanagi, Holographic entanglement entropy, Lect. Notes Phys. 931, 1 (2017).

[5] L. Susskind, Entanglement is not enough, Fortschr. Phys. 64, 49 (2016)

[6] R. Jefferson and R. C. Myers, Circuit complexity in quantum field theory, J. High Energy Phys. 10 (2017) 107.

[7] A. R. Brown, D. A. Roberts, L. Susskind, B. Swingle, and Y. Zhao, Complexity, action, and black holes, Phys. Rev. D 93, 086006 (2016).

[8] A. R. Brown, D. A. Roberts, L. Susskind, B. Swingle, and Y. Zhao, Holographic Complexity Equals Bulk Action?, Phys. Rev. Lett. 116, 191301 (2016).

[9] M. Alishahiha, Holographic complexity, Phys. Rev. D 92 , 126009 (2015).

[10] O. Ben-Ami and D. Carmi, On volumes of subregions in holography and complexity, J. High Energy Phys. 11 (2016) 129.

[11] D. Carmi, R. C. Myers, and P. Rath, Comments on holographic complexity, J. High Energy Phys. 03 (2017) 118.
[12] S. J. Zhang, Complexity and phase transitions in a holographic QCD model, Nucl. Phys. B929, 243 (2018).

[13] S. J. Zhang, Subregion complexity in holographic thermalization with dS boundary, Eur. Phys. J. C 79, 715 (2019).

[14] B. Chen, W.-M. Li, R.-Q. Yang, C.-Y. Zhang, and S.-J. Zhang, Holographic subregion complexity under a thermal quench, J. High Energy Phys. 07 (2018) 034.

[15] P. Roy and T. Sarkar, On subregion holographic complexity and renormalization group flows, Phys. Rev. D 97, 086018 (2018).

[16] R Fareghbal and P Karimi, Complexity growth in flat spacetimes, Phys. Rev. D 98, 046003 (2018).

[17] M. Alishahiha, A. F. Astaneh, M. R. Mohammadi Mozaffar, and A. Mollabashi, Complexity growth with Lifshitz scaling and hyperscaling violation, J. High Energy Phys. 07 (2018) 042.

[18] M. Alishahiha, K. B. Velni, and M. R. Mohammadi Mozaffar, Subregion action and complexity, Phys. Rev. D 99, 126016 (2019).

[19] S. A. Hosseini Mansoori, V. Jahnke, M. M. Qaemmaqami, and Y.D. Olivas, Holographic complexity of anisotropic black branes, arXiv:1808.00067.

[20] H. Ebrahim, M. Asadi, and M. Ali-Akbari, Evolution of holographic complexity near critical point, J. High Energy Phys. 09 (2019) 023.

[21] O. Andreev and V. I. Zakharov, Heavy-quark potentials and AdS/QCD, Phys. Rev. D 74, 025023 (2006).

[22] M. Ali-Akbari and M. Lezgi, AdS/QCD, entanglement entropy and critical temperature, Phys. Rev. D 96, 086014 (2017). 
[23] O. Andreev and V. I. Zakharov, The spatial string tension, thermal phase transition, and AdS/QCD, Phys. Lett. B 645, 437 (2007).

[24] O. Andreev, $1 / \mathrm{q}^{2}$ Corrections and gauge/string duality, arXiv:hep-th/0603170.

[25] J. M. Maldacena, Wilson Loops in Large N Field Theories, Phys. Rev. Lett. 80, 4859 (1998).
[26] M. Jonker, Entanglement entropy of coupled harmonic oscillators, Library Master's/Bachelor's theses, studenttheses.library.uu.nl.

[27] The temperature dependence of complexity for mixed states has been discussed in Ref. [28].

[28] C. A. Agón, M. Headrick, and B. Swingle, Subsystem complexity and holography, J. High Energy Phys. 02 (2019) 145. 